\title{
KATHARINA LORENZ
}

(Nottingham)

\section{Der Große Fries des Pergamon-Altars Die narratologische Kategorie Metalepse und die Analyse von Erzählung in der Flächenkunst}

Die Metalepse ist als narratologische Kategorie für die Analyse literarischer Texte entwickelt worden. ${ }^{1}$ Doch schließt dies ihre Anwendung im Bereich der Bildwissenschaften keineswegs aus - ganz im Gegenteil, denn gerade metaleptische Formen der Inhaltsübermittlung haben in Texten häufig eindeutig visuellen Charakter. Und so scheint es im Umkehrschluss deshalb auch durchaus angebracht, Bilder im Blick auf metaleptische Funktionsweisen zu analysieren.

Wesentliches Merkmal der Metalepse ist die Überwindung bzw. die Dekonstruktion unterschiedlicher Erzählebenen. Diese Auflösung wird dadurch erreicht, dass einzelne Elemente bzw. Figuren einer Erzählung ihre jeweilige Erzählebene verlassen - entweder innerhalb der Erzählung, oder aber auch hin zum externen Leser. Dieses Überschreiten wird jeweils durch eine bestimmte Aktion oder durch einen Sprechakt realisiert.

In der Metalepse erfahren die Erzählebenen also eine klare Korporalisierung - im Akt des Überschreitens materialisieren sie sich, und dieser Prozess verleiht ihnen durchaus visuelle Qualität: Die Erzählung erhält Räumlichkeit und Tiefe. Zugleich ist es gerade dieses Paradoxon, dass die Leser der Erzählebene in dem Moment gewahr werden, in dem sie überwunden wird, zugleich aber weiterhin fassbar bleibt, das der Metalepse solche Kraft verleiht - Kraft sowohl dazu, die Rezipienten zu verwirren, im gleichen Atemzug aber auch, oder besser: gerade dadurch auch die Erzählung ihrem Publikum näherzubringen.

Metaleptisches Erzählen ist von der Verortung der Erzählstimme(n) und den Erzählebene(n) abhängig. Zur Lokalisierung der Metalepse ist es deshalb notwendig, die Ausgangsebene bestimmen zu können, von der die Hierarchie der narrativen Ebenen innerhalb der Geschichte konstruiert

1 Genette (1983) 234-237; Genette (2004); de Jong (2009). - Für den Bereich der Bildwissenschaft vgl. auch Kemp (1989). 
wird - beispielsweise einen Ich-Erzähler. Lassen sich aber ohne eine solche Erzähl-Hierarchie Übertretungen derselben nicht bestimmen, wirft dies in Bezug auf die Analyse metaleptischer Strukturen in Bildern eine zentrale Frage auf: Wie soll man eine solche Erzählstimme im Bild lokalisieren, bzw. können metaleptische Mechanismen im Bild auch abseits eindeutig identifizierbarer Erzählebenen funktionieren?

Im Folgenden möchte ich diese Fragen am Fallbeispiel des Großen Frieses des Pergamon-Altars und im Vergleich mit weiteren antiken Bildwerken der Flächenkunst sowie der Rundplastik verfolgen. Meine These ist dabei, dass sich metaleptische Diegesen an diesen Monumenten im und durch den Raum - in den Kontaktzonen zwischen Monument und Betrachter - vollziehen, und dass diese metaleptischen Mechanismen durch weitere modale Elemente, beispielsweise die Kombination von Text und Bild, ausgeweitet werden können.

Versteht man Bilderzählung als ein Phänomen des Raumes, dann löst sich das Problem, wie in einem Bild Erzählstimmen ausgebildet werden können, denn diese lassen sich dann als jene Verbindungen zwischen Bild und Betrachter verstehen, die im und durch den Raum ermöglicht werden. Indem eine metaleptische Perspektive aber gerade diese Verräumlichung von Bilderzählung ins Zentrum stellt und mit ihr besonderes Gewicht auf die Analyse der Interaktionsbeziehung zwischen Bildwerk und Betrachter legt, wird es auch möglich, die aus der metaleptischen Analyse gewonnenen Ergebnisse für eine weiter reichende Interpretation der historischen Übermittlungsstrategien im Medium des Bildes nutzbar zu machen.

\section{Die Metalepse in der antiken Bildkunst:}

Die spätklassischen Vasenbilder

Am Beispiel spätklassischer Vasen aus dem Kreis des Meidias-Malers habe ich an anderer Stelle zu zeigen versucht, dass die Kategorie Metalepse für eine Anwendung im Bereich der Bildkunst Modifikationen verlangt (Abb. 1). ${ }^{2}$ Auf Gefäßen wie beispielsweise der namensgebenden Hydria des Meidias-Malers in London werden durch die spezifische Bildanlage und durch die Kombination von Bild und Text auf dem Bildträger bestimmte Betrachterreaktionen eingefordert. ${ }^{3}$ Diese Aufforderung zur Interaktion fungiert als medium-spezifische Erzählstimme.

2 Lorenz (2007); Lorenz [in Vorbereitung].

3 Rotfigurige Hydria, London, British Museum E224, ca. 420 v. Chr. Corpus Vasorum Antiquorum London, British Museum 6: 6-7, Taf. 91-92; ARV² 1313.5, 1690; Para 477; Add $^{2}$ 361; Burn (1987) 77, Taf. 1-9; Camponetti (2007) 17-45. 


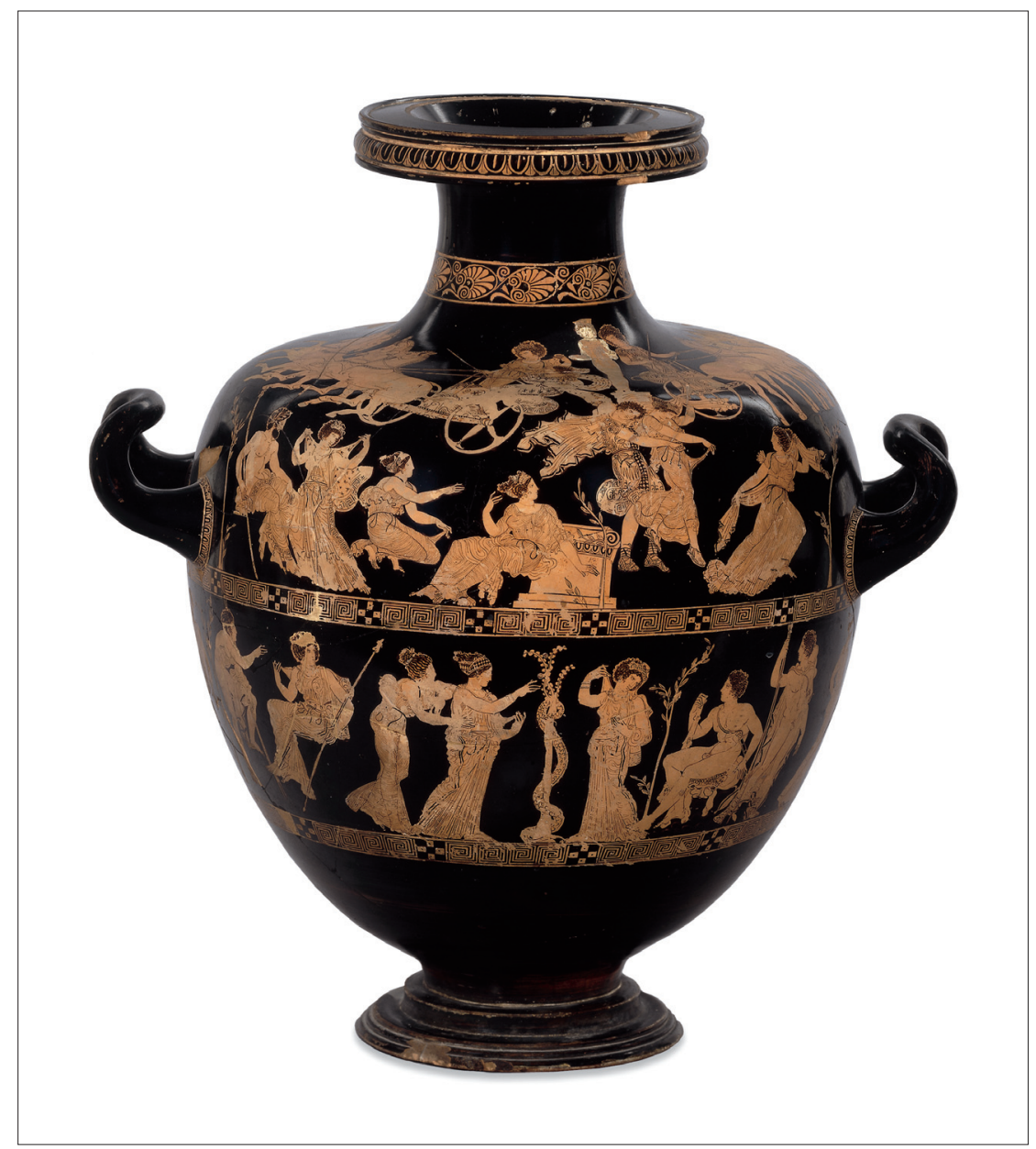

Abb. 1: Die rotfigurige Hydria des Meidias-Malers zeigt zwei unterschiedlich gestaltete Bildbereiche. London, British Museum E224, ca. 420 v. Chr. Foto: British Museum.

Die formale Bildanlage der Londoner Hydria fordert ein, dass sich die Betrachter immer wieder neu zum Gefäß positionieren, denn es zeigt drei unterschiedliche Themen. Im oberen Gefäßbereich sitzt ein frontal ausgerichtetes Panoramabildfeld, das den Raub der Leukippiden in einer dezentralisierten Komposition und mit zahlreichen Nebenfiguren zeigt. Der untere Teil ist mit einem Bildfries dekoriert, der das gesamte Gefäß umläuft. Hier ist vorn Herakles im Garten der Hesperiden zu sehen, und hinten erscheinen parataktisch komponierte Gruppen von Frauen. 
Diese einzelnen Bereiche des Gefäßes, die erzählerisch nicht miteinander in Zusammenhang stehen, sind dennoch in verschiedener Weise aufeinander bezogen. So lässt sich etwa zwischen den Frauen im hinteren Teil des Bildfrieses und zwei Figuren im oberen Panoramabild ein Bezug herstellen, der die beiden Gefäßteile im Diskurs über weibliches Rollenverhalten verbindet: Eriphyle, eine der beiden Leukippiden, und ebenso Chryseis, eine der Nebenfiguren, gleichen den Frauen im unteren Bildfries ikonographisch deutlich. Zum anderen werden aber durch die Verteilung der Bildfelder auf dem Gefäßkörper eigentlich zusammengehörige Erzählbereiche aufgebrochen, weil sie nicht auf einen Blick in ihrer Gesamtheit sichtbar sind. Dies gilt hier insbesondere für die raumgreifende Darstellung vom Raub der Leukippiden, die neben der konkreten Situation des Raubes weitere Aktionsbereiche umfasst. Um die Geschichte, wie sie im Bild dargestellt ist, zu erkunden, muss der Betrachter sich bzw. das Gefäß bewegen.

Damit werden die Darstellungen partitioniert, und zwar in einer Weise, die diese einzelnen Erzählrahmen zu eigenständigen Erzählebenen werden lässt. Und in der Bewegung des Gefäßes können dann Bezüge zwischen diesen Ebenen hergestellt werden - und zwar auch zwischen Teilen des Gefäßes, die ansonsten nichts miteinander zu tun haben, aber nun gemeinsam sichtbar sind. Im Fall der Londoner Hydria bedeutet dies etwa, dass der Betrachter nahtlos von einer mythischen Erzählung - dem Raub der Leukippiden - hinein in einen aktuellen Diskurs zum Verhalten athenischer Frauen wechseln kann, wie er im Rückteil des unteren Bilderfrieses dargestellt ist. Diese Form der Diegese lässt sich als metaleptisch klassifizieren - eine Metalepse, die hier möglich wird, weil der Gefäßkörper qua seiner räumlichen Ausdehnung als „Erzählkörper“ fungiert: Er übernimmt die Rolle eines extradiegetischen Erzählers, führt die Betrachter durch das Bild oder die Bilderrahmen und ersetzt so die Sprechakte eines Textes.

Zudem können einzelne der dargestellten Charaktere mit Hilfe von Textbeischriften ihren Bezugsrahmen wechseln - sie agieren so sowohl als Figuren einer spezifischen Erzählung als auch als allgemeingültige, aktions- und qualitätsbeschreibende Personifikationen. Im Fall der Londoner Hydria gilt dies insbesondere für die Figur der Eriphyle. ${ }^{4}$ Sie erscheint in der Rolle einer der Leukippiden im oberen Bildfeld; doch ist ihr Name ansonsten für diese Rolle nicht attestiert. In seiner wörtlichen Bedeutung aber verwandelt der Begriff die Leukippidin in eine Personifikation, welche die positiven Konnotationen einer Phyle feiert - jener Verwaltungseinheit also, die den Kern des athenischen Gemeinwesens ausmacht. Dies

$4 \quad$ Lorenz (2007) 135-143. 
wiederum macht aus der Darstellung des Leukippidenraubes auch eine Geschichte über das attische Staatswesen.

Dieser fließende Wechsel zwischen narrativen und deskriptiven Qualitäten einzelner Figuren im Bild ist vielleicht am besten als modale Metalepse zu titulieren. Literaturwissenschaftlich-narratologisch gesprochen müsste man hier wohl korrekter von einem Perspektivwechsel bzw. einem Wechsel der Erzählstimme sprechen. Das Beispiel der Londoner Hydria des Meidias-Malers zeigt jedoch, dass in diesem Punkt im Zusammenspiel von Materialträger und Namensbeischriften eine Struktur erzeugt wird, die jenem Überschreiten unterschiedlicher Erzählebenen äquivalent scheint, das eine Metalepse charakterisiert. Im Unterschied zu der durch den Gefäßkörper erzeugten Situation der ontologischen Metalepse - eine Metalepse, die den Handlungs- bzw. Darstellungsraum überwindet kann dies wohl am besten als eine epistemologische Metalepse bezeichnet werden - als eine Metalepse, die den Wechsel in einen neuen fiktionalen Bezugsrahmen beschreibt. ${ }^{5}$

Das Beispiel der spätklassischen Vasenbilder zeigt, dass hier die Form des Gefäßes als Motor für die Erzählung neu entdeckt wird - eine Funktion, welche die Gefäßkörper sicher immer hatten, die aber vorher kaum derart weit reichend ausgenutzt wurde. Und gerade diese Führungsfunktion des Gefäßkörpers ist es, welche die Erzeugung metaleptischer Situationen erlaubt; und zugleich stellt diese Führungsfunktion ganz allgemein eine entscheidende Errungenschaft in einem Bildmedium dar, denn sie macht Erzählung im Bild überhaupt erst möglich.

\section{Die Metalepse in der antiken Bildkunst: Die Großplastik}

Das Beispiel der Vasen zeigt, wie wichtig die Rolle des Materialträgers als Erzählkörper zur Erzeugung metaleptischer Situationen ist. Blickt man auf die Großplastik, so gestaltet sich hier die Situation zweifach anders. Zum einen gilt dies in Bezug auf die Aktivierung durch den Betrachter bzw. die Betrachteraktivierung durch das Bild: Während es bei den Vasen in Anbetracht ihrer handhabbaren Größe verhältnismäßig leichtfällt, durch Drehung des Gefäßes bzw. des Körpers die verschiedenen Ebenen

5 Zur Definition der beiden Typen von Metalepse s. Mahne (2007) 76, 102. - Der Typ der epistemologischen Metalepse lässt sich nicht mit jenem der diskursiven bzw. rhetorischen Metalepse gleichsetzen, sondern steht parallel zu diesen Formen. - Zur rhetorischen Metalepse: Ryan (2005); Ryan (2006) 204-230, 246-8. Zur diskursiven Metalepse: Fludernik (2003). 
der Transmission zu erkunden, ist dies bei Skulpturen einer bestimmten Größe oder solchen, die an Gebäuden verankert sind, nicht möglich.

Es stellt sich die Frage, ob dieser Unterschied in Bezug auf die Interaktionsaktivierung auch Folgen für den Einsatz und das Funktionieren metaleptischer Erzählelemente hat: Handelt es sich bei den unterschiedlichen Rezeptionssituationen nur um eine quantitative Differenz (der Weg um eine Skulpturengruppe oder um einen Tempel mit Architekturdekoration ist länger als jener um eine Hydria), oder geht damit auch eine qualitative Verschiebung in der Funktionsweise metaleptischer Erzählung einher?

Zum anderen trägt gerade die Großplastik gewissermaßen a priori metaleptisches Potential, denn sie präsentiert etwas, das eigentlich nicht zu der Daseinsebene des Betrachters gehört - einen Menschen, ein Ereignis - in einer Art, als wäre es Teil dieser Daseinsebene, auch wenn einzelne Monumente dies dann ganz unterschiedlich einlösen können. Richard Neer hat kürzlich umfassend dargelegt, wie die bildhauerische Gestaltung klassischer Skulptur zu Seherlebnissen beitragen kann, welche die Statuen fest in der Daseinsebene der Betrachter verankern und trotzdem aber auch Spuren davon enthalten, dass sie dort eigentlich nicht wirklich hingehören. ${ }^{6}$ Jene Form der gleitenden Metalepse, die sich in der antiken Literatur verschiedentlich attestieren lässt, scheint damit auch der Großplastik als wesentliches Gestaltungsprinzip zugrunde zu liegen. ${ }^{7}$ Neer zeigt dies eindrücklich an Beispielen der Frühklassik; und auch wenn er die dort beobachteten Phänomene nicht als Metalepse benennt, so ist doch die in den Skultpuren zum Einsatz gebrachte Rhetorik, die er eindrücklich herausarbeitet, inhärent metaleptisch.

Betrachten wir das Beispiel der Tyrannentöter. Zunächst ist aus metaleptischer Perspektive relevant, dass die beiden, Harmodios und Aristogeiton, Seite an Seite gegen einen imaginären Gegner anstürmen. Dieses Aussparen des Ziels der hier zur Schau gestellten Dynamik perpetuiert die Aktion der beiden: Sie repräsentieren kein historisches Einzelereignis, sondern einen ideologischen Zustand. Gleichzeitig aber wird der Betrachter durch diese spezifische Komposition auch in die Rolle des Reaktanten gesetzt, denn der Betrachter ist sowohl Publikum der beiden Tyrannentöter, zugleich aber durchaus auch potentielles Ziel ihrer Tat, denn ansonsten ist ja kein Opfer dargestellt. Und somit treffen hier die Daseinsebene des Artefakts und jene des Betrachters in einem Akt der ontologischen Metalepse aufeinander.

6 Neer (2011) 57-69.

7 Zum gleitenden Charakter von Metalepse in der Literatur s. in diesem Band 2. 
Ein weiteres formales Element rückt die Tyrannentöter dem Betrachter näher: Durch die perspektivische Verkürzung der Rücken- bzw. Seitenansicht erscheinen ihre Oberkörper dem Betrachter angenähert, ohne dies eigentlich wirklich physisch zu sein. ${ }^{8}$ Neer interpretiert diesen Sachverhalt - zugleich auch mit Bezug auf das Epigramm des Simonides, das die Basis der originalen Statuengruppe auf der Athener Agora schmückte - als Strategie, um die beiden Figuren einem Lichtstrahl gleich erscheinen zu lassen, der mit Wucht auf den Betrachter trifft, um ihn in Staunen zu versetzen. ${ }^{9}$ Auch dieses Charakteristikum - die formale Gestaltung, die darauf zielt, die Skulpturen als lebendigen Teil der Realität zu bestätigen - ist als eine ontologische Metalepse zu werten.

Nochmals verstärkt begegnet uns dieses Phänomen in der Kunst des Hellenismus. In den für die Plastik des 3. Jahrhunderts so charakteristischen mehransichtigen Gruppen wie etwa dem Gallier Ludovisi oder dem Farnesischen Stier begegnen uns Werke, die nicht nur dreidimensional im Raum positioniert sind, sondern vom Betrachter auch eine dezidiert dreidimensionale Betrachtungsweise einfordern. ${ }^{10}$ Diese Statuengruppen erschließen sich nicht nur von ein oder zwei Seiten, sondern nur in Rundumsicht des gesamten Monuments, wobei sich dem Betrachter dabei je nach Ansicht auch sich widersprechende Aussagen über das Monument eröffnen können; andersherum aber können sich so natürlich andere Aspekte der Monumente immer wieder dem Betrachter verschließen. ${ }^{11}$ Dazu kommt, dass im Hochhellenismus die Statuen direkter mit ihrer Umgebung verzahnt werden, so als wären sie tatsächlich Teil von dieser. ${ }^{12}$

Die hellenistische Literatur liefert verschiedene Beschreibungen solcher Statuen, und dabei wird als Grundthema stets ihre große Wirklichkeitsnähe hervorgehoben. ${ }^{13}$ Das wohl eindrücklichste Beispiel findet sich bei Herodas, der den Besuch zweier Frauen in einem Heiligtum und ihre Kommentare über die dort ausgestellten Skulpturen schildert: ${ }^{14}$

\footnotetext{
$8 \quad$ Neer (2011) 78.

9 Neer (2011) 81-83.

10 Zum Gallier Ludovisi: Kunze (2002) 40-43; Marvin (2002). - Zum Farnesischen Stier: Kunze (1998); Kunze (2002) 25-38.

11 Zur Kompositionsweise hellenistischer Gruppen zuerst Krahmer (1923/24); Krahmer (1925); s. auch Künzl (1968). - Mit kritischer Diskussion nun Kunze (2002) 12-20, 229-238. - Zum Verhältnis dieser Statuengruppen zu Erzählformen in der gleichzeitigen Literatur s. Zanker (2004) 72-103.

12 Kunze (2002) 232-239.

13 Zanker (1987) 39-112; Manakidou (1993); Kunze (2002) 233-234. - Vgl. dazu auch in diesem Band Baumann: 278.

14 Herodas 4, bes. 4,27-38; s. Zanker (2009) 98-113.
} 
Kynno: „Siehst Du, meine Liebe, das Mädchen zu dem Apfel hinaufblicken? Würdest Du nicht sagen, dass sie - sollte sie den Apfel nicht erreichen - auf der Stelle in Ohnmacht fallen würde?"

Kokkale: „Und der alte Mann, Kynno - oh, bei den Moiren, wie der kleine Junge die Gans würgt!“

Kynno: „Wäre es nicht Stein vor unseren Füßen, so - wenigstens - würdest Du doch sagen, die Statue sei im Begriff zu sprechen. Oh, mit der Zeit werden es die Menschen schon fertigbringen, den Steinen Leben einzuflößen.“

Vergleicht man die Wirkung dieser mehransichtigen Gruppen mit der Funktionsweise der spätklassischen Vasen und klassischer Skulpturen wie etwa den Tyrannentötern, so zeigt sich einmal mehr, dass die Aktivierung durch den Betrachter durchgehend entscheidend für die Schaffung metaleptischer Erzählsituationen ist.

Der Große Altar von Pergamon - Metalepse in der Flächenkunst

Die Flächenkunst - die Darstellung im Relief - nimmt eine Zwischenstellung zwischen der zweidimensionalen Malerei und der Großplastik ein. Flächenkunst funktioniert gerade nicht dreidimensional bzw. nicht so eindeutig dreidimensional wie die Großplastik, denn zumeist wird sie erst dadurch, dass sie an einem Bau verankert ist, vollständig dreidimensional. Andererseits aber sind diese Reliefs trotzdem dreidimensionaler als die Vasenmalerei und - wiederum anders als die Vasen - lassen sich im Allgemeinen nicht derart leicht bewegen wie diese. So stellt sich hier die Frage nach den Möglichkeiten metaleptischen Erzählens im Medium des Bildes im und durch den Raum nochmals neu.

Der Große Altar von Pergamon bietet sich als Fallstudie für eine derartige Untersuchung an. Der Bau wurde auf einer der Terrassen des Pergamener Burgbergs in der ersten Hälfte des 2. Jahrhunderts v. Chr. wahrscheinlich unter der Herrschaft des Attalidenkönigs Eumenes II. (197-159 v. Chr.) errichtet. ${ }^{15}$ Zwei Aspekte des Altars sind von besonderem Interesse in Bezug auf die Frage nach metaleptischen Mechanismen im Medium des Bildes. Dies ist zum einen seine Form und Position; zum anderen seine Dekoration, und insbesondere der Große Fries mit der Darstellung einer Gigantomachie, der den Bau außen umläuft. ${ }^{16}$

15 Weder der genaue Entstehungszeitpunkt noch Anlass und Funktion des Altars sind abschließend geklärt. Zur Diskussion zuletzt: Kästner (2011). S. auch: De Luca/Radt (1999) XV-XIX; Scholl (2009).

16 Zur Dekoration des Altars im Überblick: Winnefeld (1910); Kähler (1948); Queyrel (2005); Massa Pairault (2007a). 
Mit seiner П-Form setzt sich der Altar deutlich von früheren Altarbauten ab. ${ }^{17}$ Zugleich aber, und im Verbund mit der Positionierung des Gebäudes auf der Altarterrasse, wird so erreicht, dass Besucher möglichst lange mit den Außenwänden des Monuments - und insbesondere mit dem dort angebrachten Großen Fries - in Interaktion stehen: Betritt man die Altarterrasse, so hat man zunächst die Oststeite, die Rückseite des Gebäudes, vor sich; d.h. der gesamte Komplex muss umschritten werden, um zur Freitreppe zu gelangen, die hinauf zum Altarhof führt. Und durch die П-Form folgt der Fries entlang der inneren Risalitwände dem Besucher sogar die Treppen hinauf.

\section{Der Große Fries und das metaleptische Erzählen}

Die architektonische Form des Altarbaus und die Gestalt des Großen Frieses lassen diesen den Betrachtern auf besondere Weise ins Auge stechen. Der Fries ist Teil des Podiums des Altarbaus. Er sitzt etwas mehr als $2 \mathrm{~m}$ über dem Boden und misst 2,3 $\mathrm{m}$ in der Höhe und ca. $113 \mathrm{~m}$ in der Länge. ${ }^{18}$ Die Figuren des Frieses waren im ursprünglichen Zustand bemalt und der Bildhintergrund in einem dunklen Blau ausgeführt. ${ }^{19}$ Zudem waren die Szenen mit zahlreichen Metallappliken weiter ausgeschmückt. Zu diesen formalen Besonderheiten kommt hinzu, dass die im Fries dargestellte Gigantomachie sich bereits in ihrer Anlage deutlich von anderen derartigen Darstellungen unterscheidet und auch deshalb ins Auge gefallen sein dürfte. ${ }^{20}$

Zunächst ist hier die dezidierte Benennung der Figuren zu verzeichnen: Alle Akteure trugen eine Namensbeischrift, jene für die Götter waren am oberen Deckgesims angebracht, die für die Giganten am unteren Fußprofil. ${ }^{21}$ Diese Identifizierungshilfe erscheint vor dem Hintergrund der anderen Besonderheit des Frieses durchaus sinnvoll, nämlich der ungewöhnlich hohen Zahl der Figuren insgesamt, die kein Betrachter so je in einer Gigantomachie gesehen haben dürfte, und dazu noch der deutlichen Überzahl der Giganten gegenüber den Göttern, die zumindest zum Teil durch ihre Attribute identifizierbar sind: Auf Seiten der Götter sind 49 Kämpfer erhalten, auf jener der Giganten 50. Dazu ist noch bemerkens-

17 Zur architektonischen Form des Baus und seinem Verhältnis zu anderen Altarbauten s. Stähler (1978); Hoepfner (1989); Hoepfner (1993); Linfert (1995) 131-146; Scholl (2011).

18 Zu den Maßen s. Kästner (2011) 207-208.

19 Zum Verhältnis von Figur und Raum am Großen Fries s. Kähler (1948) 88-96.

20 Zu den Darstellungen der Gigantomachie s. LIMC IV 1988 s. v. Gigantes [F. Vian /M. B. Moore]; s. auch: Vian (1951).

$21 \mathrm{Zu}$ den Inschriften s. Kästner (1994). 


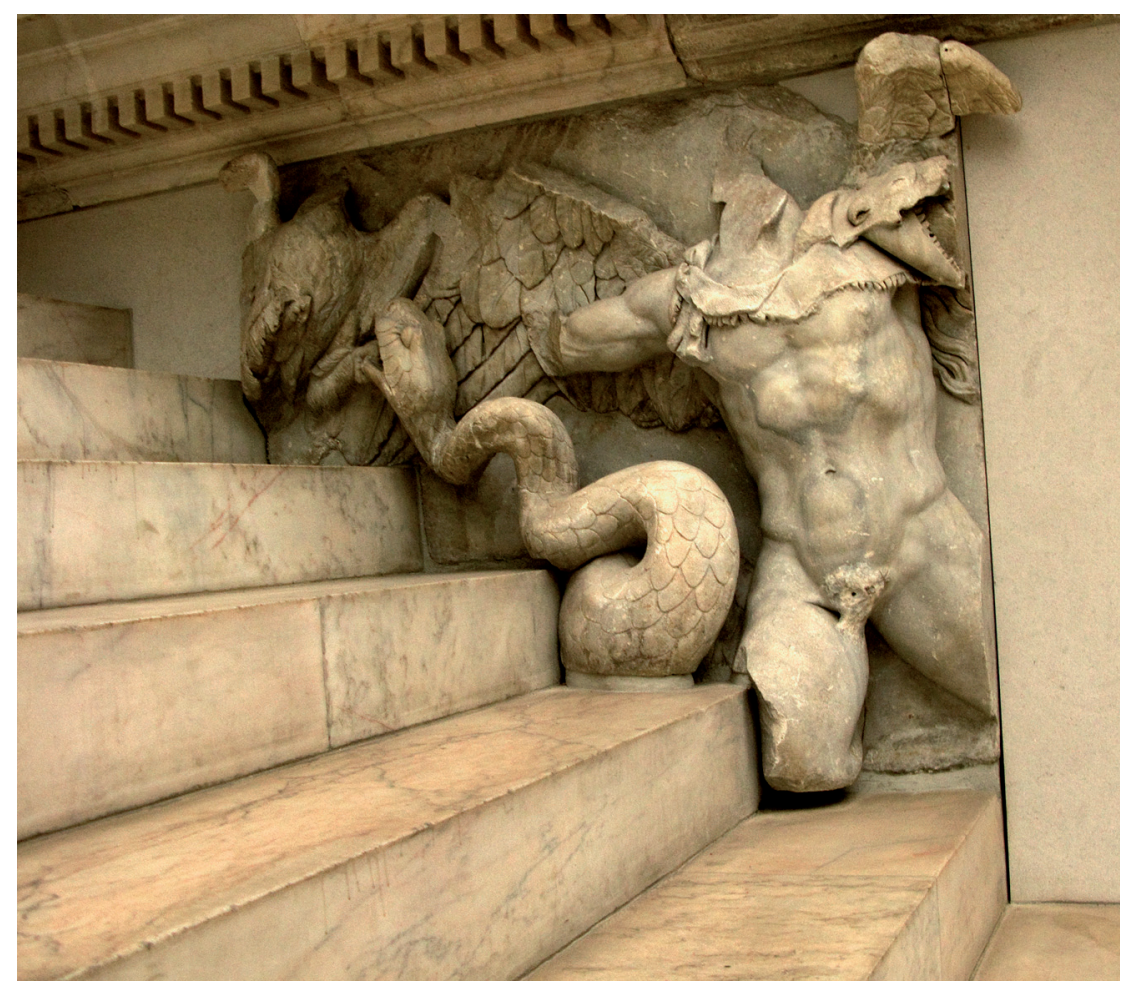

Abb. 2: Am Südrisaliten ist der Körper des Giganten Bronteas zum Teil auf der Freitreppe des Altars platziert. Foto: Autor.

wert, dass die Götter in Familiengruppen und Wirkungsbereichen über den Fries verteilt sind: Die Olympier kämpfen im Osten; im Süden die Titanen; im Norden das Geschlecht der Nyx; und um den N/W-Risaliten das Geschlecht des Pontos. ${ }^{22}$

Eine eindeutig metaleptische Darstellungssituation findet sich an den Risalitinnenseiten, die der Treppe nach oben zum Altarhof folgen. Sowohl am Nord- wie am Südrisaliten quellen hier, in den sich verjüngenden Zwickeln des Frieses, Gigantenleiber und Schlangenbeine auf die Treppenstufen (Abb. 2). Im Süden ist es der Körper des Giganten Bronteas („Donner"), der auf den Stufen in die Knie gebrochen ist, den Körper frontal dem Betrachter zugewandt, mit dem Kopf aber nach rechts die

22 Zur Verteilung der Götter auf dem Fries: Simon (1975). Mit interessanten Gegenargumenten: Pfanner (1979). 


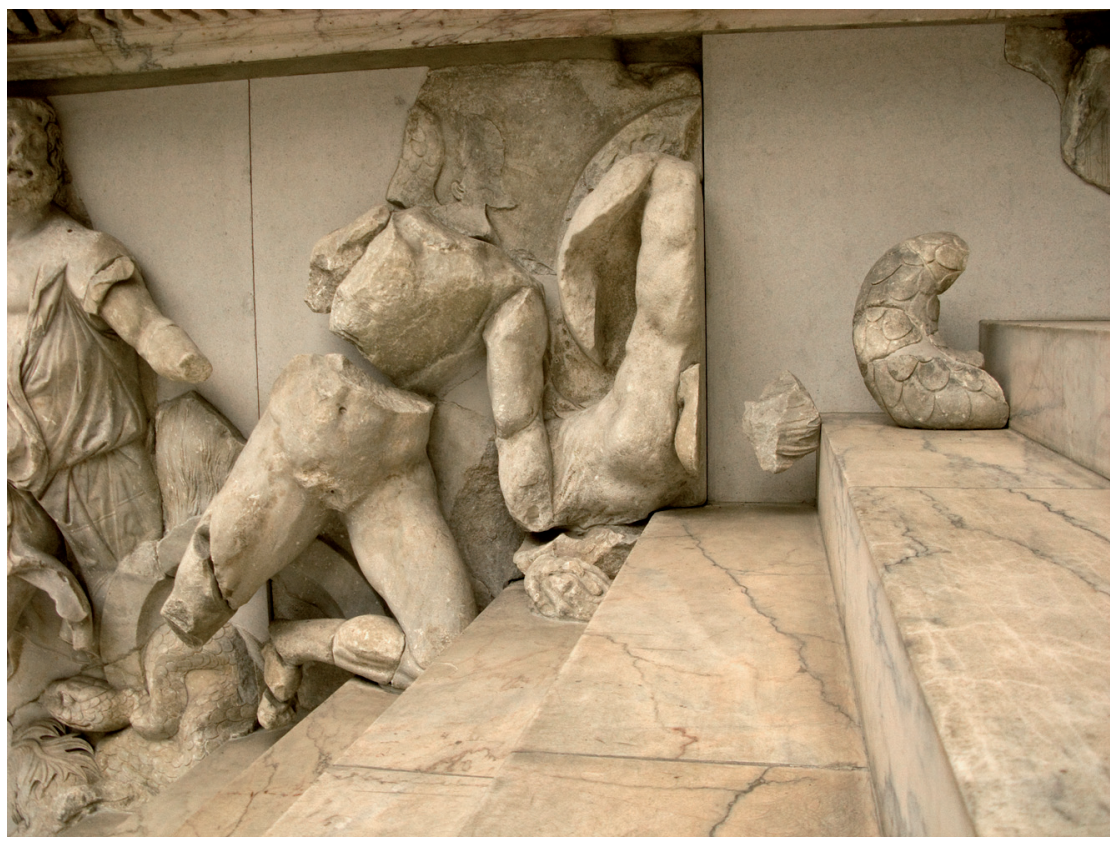

Abb. 3: Am Nordrisaliten weichen die Gegner von Thetys und Okeanos auf die Treppe aus. Foto: Autor.

Treppe hinabblickend und wohl auch in diese Richtung gegen eine der Nymphen kämpfend. ${ }^{23}$ Sein linkes Bein endet in einem Schlangenkörper, und dieser schraubt sich nach links die Stufen herauf, um dort den Adler des Zeus zu attackieren, der aber seinerseits siegesgewiss den Unterkiefer des Reptils in einer seiner Klauen hält.

Im Norden sind es die beiden Gegner von Thetys und Okeanos, die wiederum aus dem Fries und auf der Treppe agieren (Abb. 3): Der vordere, gänzlich menschlich gebildete Gigant ist frontal gesehen. Er ist in der Flucht treppauf gezeigt. Mit dem linken Knie ist er bereits auf die Stufen gebrochen, greift aber noch mit seiner Linken nach einem Felsbrocken, ebenfalls auf den Stufen positioniert, vielleicht um ihn mit letzter Kraft gegen den mächtigen Okeanos zu schleudern. Sein Kamerad befindet sich weiter oberhalb und ist im Rückenakt auf den Treppenstufen sitzend gezeigt, sich mit einem Schild gegen den Angriff von unten schützend. Sein linkes Schlangenbein läuft neben Okeanos aus, leistet jedoch keine

$23 B P O$ ist inschriftlich gesichert, s. Kästner (1994) 126. 
Gegenwehr; sein rechtes könnte im Treppenzwickel ähnlich wie Bronteas gegenüber gegen den dort positionierten Adler des Zeus gekämpft haben.

Die Wirkung dieser aus dem Fries heraus und auf die Treppe sich ergießenden Gigantenleiber muss im Originalzustand noch durch die Bemalung sowohl der Figuren als auch des Frieshintergrunds betont gewesen sein, und andererseits dadurch, dass die Treppenstufen selbst marmorgrundig belassen waren. Diese distinkte farbliche Trennung betonte nun andersherum natürlich umso mehr den Akt des Übertritts der Giganten aus ihrer Sphäre hinein in jene der Betrachter.

Fragt man nach dem Resultat, der inhaltlichen Wirkung dieses Arrangements, so scheint die Komposition in den Treppenzwickeln zunächst ganz linear aus der Anlage des Frieses insgesamt entwickelt. Die Gigantomachie brandet wie in einer Momentaufnahme in wildem Getümmel um den gesamten Altar. Und doch lässt sich an vielen Stellen des Frieses beobachten, dass die Komposition davon beherrscht wird, den Betrachter, wie weiter oben beschrieben, um das Gebäude herum und zum Aufgang zum Opferaltar zu lenken.

Gerade die Darstellungen an den Ecken können durch ihre Bewegungsausrichtung eine besondere Leitfunktion einnehmen. So begegnet man etwa Hekate am südlichen Ende des Ostfrieses kräftig zum Umbruch des Frieses hin nach Süden ausschreitend; und über Eck ist es dann Phoibe, die in Richtung Westen und zum Altaraufgang weiterführt. Ebenso ist die Komposition auf den Stirnseiten der Risaliten mit Triton im Norden und Semele im Süden in Richtung auf den Treppenaufgang hin ausgerichtet, und über Eck nehmen Nereus und Doris diese Bewegung hinauf zum Altarinnenhof auf.

Mit Blick auf dieses treppan drängende Kampfgetümmel leisten jene auf die Treppenstufen ausweichenden Giganten eine Verstärkerfunktion: Es scheint, als ob ihnen dies als einziger Ausweg gegen die heranstürmenden Götter bliebe, und damit wird ihre Niederlage durch das metaleptische Herausdrängen gewissermaßen formal besiegelt. Zugleich wird aber auch die Kraft der Götter unterstrichen, denn es ist ja jene, durch die es erst möglich wird, die Grenzen des Frieses derart aufzubrechen. Andererseits scheint im Akt der Metalepse eine weitere Ebene angerissen, und zwar dadurch, dass nur die Giganten, nicht aber die Götter auf den Treppen erscheinen. Zunächst ließe sich ja durchaus daran denken, dass durch das Herauspräparieren der Giganten - und die Situierung in der Sphäre der Betrachter Sympathie bzw. so etwas wie Mitleid für die Giganten erzeugt werden soll. ${ }^{24}$

24 Dies in Analogie zu der Lesart, die Tonio Hölscher für das Große Attalische Weihgeschenk vorschlug: Hölscher (1985). 
Doch ist das Ziel hier eine Pathetisierung ganz anderer Art. Durch die Positionierung der Gliedmaßen der Giganten auf den Stufen wird es für den Betrachter möglich, ganz handfest genau das zu tun, was auch die Götter mit ihren Gegnern betreiben: kräftig auf die Gigantenleiber zu treten, so wie es etwa auch Doris im unteren Teil der Innenseite des nördlichen Risaliten tut, oder auch Artemis am Südfries und Aphrodite am Nordfries.

So ist hier die Metalepse gewissermaßen als Einladung zum Mitkämpfen eingesetzt. Der Betrachter ist damit nicht mehr nur Zeuge des Vernichtungskampfes der Götter, sondern wird zum göttlichen Bundesgenossen. Die Folge einer derartigen Einbeziehung ist durchaus eine Realitätssteigerung der Darstellung: Sie ist nicht mehr nur entrückte Repräsentation, sondern wird zu einem tatsächlichen Bestandteil der Daseinsebene der Betrachter. Doch artikuliert sich damit zugleich auch eine Entrückung anderer Art: Während die Giganten in den Lebensbereich der Betrachter treten, werden die Betrachter gerade dadurch in den Kreis der Götter gehoben, denn sie können nun agieren wie diese.

Die Mittäterschaft, und zugleich das Element der Divinisierung, stellt sich gerade im Kontext der Gigantomachie als besonders signifikant dar. Zunächst, weil es eine Veränderung der sonst für die Darstellungen der Gigantomachie üblichen Strategien darstellt. Die Gigantomachie erfreut sich vor allem im 6. und 5. Jh. in der Vasenmalerei und in der Architekturplastik großer Beliebtheit. ${ }^{25}$ Die Darstellungen gleichen in dieser Zeit im Wesentlichen einem Hoplitenkampf: Einzelne Kampfgruppen - im Profil gesehen - treffen aufeinander. Die Gigantomachie vom N-Fries des Siphnierschatzhauses in Delphi, datiert um 525 v. Chr., bietet für die Untersuchung metaleptischen Erzählens im Bereich von Gigantomachiedarstellungen den besten Anknüpfungspunkt. ${ }^{26}$

Ähnlich wie auch am Pergamon-Altar sind hier Namensbeischriften zur Identifizierung von Göttern und Giganten eingesetzt; ${ }^{27}$ und ebenso lädt die Hauptlaufrichtung der Götter im Fries von Osten nach Westen den Betrachter dazu ein, dem Fries die Heilige Straße hinauf und zum Eingang des Schatzhauses zu folgen. Die Komposition macht die Betrachter hier zu Gefolgsleuten der Götter, jedoch nicht zu Mittätern. Der Kampf am Fries ist in sich geschlossen: Wie im Hoplitenkampf üblich

25 Für einen Überblick über die ikonographische Entwicklung s. LIMC IV 1988 s. v. Gigantes [F. Vian/M. B. Moore]; Kähler (1948) 107-109, ebenso Viant (1951). - Zur Rolle der Gigantomachie in den Bilderwelten des 6. und 5. Jhs. s. Giuliani (2000); Muth (2008) 268-328.

26 Zum Siphnierschatzhaus: Simon (1984); Neer (2001).

27 Brinkmann (1985), zum N-Fries bes. 87-105, 121-130. 
ziehen die Kampfreihen gegeneinander, und dies schließt das Einbeziehen der Betrachter aus.

Das einzige metaleptische Element, das sich am Nordfries des Siphnierschatzhauses findet, macht den Unterschied besonders deutlich. Im östlichen Drittel des Frieses wird ein Gigantenhoplit von einem der Löwen erfasst, die den Wagen der Kybele ziehen. Das Tier hält den Oberkörper seines Gegners in den Pranken und hat sich dort festgebissen. Der Gigant versucht sich mühsam - aber erfolglos - wie in einem Ringkampf aus der Umklammerung zu lösen. Die ansonsten durchgängige Profilansicht des Frieses ist hier unterbrochen, und die Gesichter des Löwen ebenso wie des Gigantenhopliten, wenn auch unter dem korinthischen Helm fast völlig verborgen, sind frontal gesehen.

Die hier gewählte Komposition, die das metaleptische Aus-dem-FriesSchauen der beiden Figuren als Schock-Effekt einsetzt, artikuliert somit effektvoll die Gewalt der Götter und ihren Erfolg. Damit ähnelt das Arrangement inhaltlich durchaus der Darstellung auf dem Großen Fries in Pergamon. Anders jedoch als dort wird der Betrachter damit nicht zur Partizipation eingeladen, er bekommt lediglich vorgeführt, wie die Götter und ihre Trabanten erfolgreich den Kampf bestehen.

\section{Metaleptische Diegesen}

Es ließe sich nun argumentieren, dass es sich bei den am Großen Fries herausquillenden Figuren lediglich um eine formale Zufälligkeit handelt, die der Tatsache geschuldet ist, dass hier eine ungewöhnliche architektonische Bauform mit einer ebenso ungewöhnlichen Friesdekoration geschmückt ist.

Das ungewöhnlich hohe Relief des Großen Frieses ist an sich bereits aufs Äußerste raumgreifend, nicht nur auf den Stufen. ${ }^{28}$ So ließe sich durchaus denken, dass die Situation auf den Treppenstufen nur eine Fortsetzung dieser Plastizität ist, die hier gezwungenermaßen auf die Fläche der Stufen ausgedehnt ist, weil in diesem Bereich des Frieses die ansonsten vom Fußprofil gebildete Standfläche wegfällt. Das Ergebnis wäre dann also nicht als bewusster Akt metaleptischer Komposition zu werten, sondern als eine durch die Architektur diktierte Unumgänglichkeit. Und die Tatsache, dass einzig Gigantenfiguren betroffen sind, ließe sich dann als schlichter Zufall werten.

28 Vgl. Kähler (1948) 84-89 mit einer Diskussion der durch das Hochrelief erzielten Realitätssteigerung. 
Dagegen lassen sich jedoch weitere Charakteristika des Baus und seiner Dekoration ins Feld führen. Und dies führt in einen Bereich, in dem metaleptische Übermittlung nun in einem deutlich weiteren Sinne verstanden werden muss, bzw. - umgekehrt - lässt uns dies nun die Skalierung unterschiedlich intensiver metaleptischer Diegesen am Großen Altar studieren.

Metaleptische Skalierungen: Der Große Fries aus der Distanz

Seine Position auf einer eigenen Terrasse auf der Pergamener Akropolis macht den Altar aus der Distanz vorzüglich sichtbar. Zudem setzt ihn sein weißer Marmor deutlich von den Substruktionsmauern der Altarterrassen ab. ${ }^{29}$ Betrachtet man die unterschiedlichen Perspektiven, aus denen der Altar für die Bewohner und Besucher Pergamons sichtbar war, lassen sich verschiedene Situationen unterscheiden, die jeweils bestimmte Betonungen auf einzelne Aspekte des Baus beobachten lassen. Und allen diesen Sichtperspektiven ist gemeinsam, dass sie jeweils die räumliche Beziehung zwischen Betrachter und Erzählung neu verhandeln, und damit auch die Positionierung des Betrachters im Verhältnis zu den unterschiedlichen Ebenen der Erzählung - und genau dies erzeugt eine jeweils unterschiedliche metaleptische Sogwirkung, die insgesamt dann wiederum die Erlebbarkeit des Monuments und der dargestellten Erzählung bestimmt.

Zunächst ist hier die Ansicht aus der Distanz zu bedenken: Für Betrachter, die von SW die Stadt betreten, stellt sich der Altar als eigenständiger Teil der Akropolis dar, auf seiner eigenen Terrasse situiert. ${ }^{30}$ Es muss unklar bleiben, ob bzw. wie genau einzelne Elemente der Friesdarstellung aus solch einer Fernsicht wahrgenommen werden konnten.

Für jene die Akropolis hinaufsteigenden Betrachter eröffnet sich von der Oberen Agora eine interessante Ansicht auf den Großen Fries. ${ }^{31}$ Aus dieser Perspektive erscheint der Altar nicht mehr als eigenständiges Monument, sondern vielmehr als Basis für den Tempel der Athena, der hinter ihm auf der darüberliegenden Terrasse des Athena-Heiligtums positioniert ist. $^{32}$

29 Scholl (2011) 73.

30 Schraudolph (2007) 198.

31 Zur Umstrukturierung der Oberen Agora unter Eumenes II. siehe Rheidt (1992), bes. 266-269. Vgl. La Rocca (1998); Massa Pairault (2007b) 3-6.

32 Die Annäherungssituation ist jener im Athena-Heiligtum von Lindos vergleichbar, dazu Hoepfner (1989) 622-624. 
Der Altar wird so konkret in die inhaltliche Ausrichtung der Oberen Agora selbst eingepasst. Der Marktplatz bildet seit etwa 220/10 das städtische Zentrum Pergamons, und er ist insbesondere durch den von Attalos I. gegründeten Tempel des Zeus Soter charakterisiert, ${ }^{33}$ und zudem durch Weihungen der königlichen Familie, die ihre militärischen Erfolge zelebrierten. ${ }^{34}$ Somit wird hier über die Sichtachse auch eine inhaltliche Brücke zwischen den drei Terrassen geschlagen - der Oberen Agora, dem Großen Altar und dem Heiligtum der Athena Nikephoros -, die die Gesamtanlage in den Dienst attalischer Staatsideologie stellt, und zwar ganz spezifisch mit Blick auf die Qualität „Sieghaftigkeit“. ${ }^{35}$

Der Große Altar ist in diesem Kontext zunächst schlicht nur architektonisch eindrucksvolle Basis für den sich darüber erhebenden AthenaTempel. ${ }^{36}$ Von den Weihungen im westlichen Teil der Oberen Agora beträgt die Distanz zur S-Flanke des Altares ca. 50 m, von demselben Punkt hinauf zum Tempel der Athena Polias auf der Athena-Terrasse ca. 170 m. Betrachtet man nun die S-Seite des Großen Frieses, so ist hier eine besondere Form der Komposition festzustellen, die sich von den anderen Seiten absetzt, und die aus der Distanz von der Oberen Agora durchaus wahrzunehmen gewesen sein dürfte.

Die S-Seite des Großen Frieses zeichnet sich durch eine Komposition aus, in der sich zentriert-arrestierende und leitende Elemente abwechseln. In der östlichen Hälfte lässt sich an den Figuren deutlich ein Bewegungsdrang in Richtung Westen beobachten, zunächst primär in der Figur der Phoibe artikuliert, die in wehendem Gewand und mit einer großen Fackel bewaffnet schwungvoll in Richtung Westen vorstößt. Weiter links perpetuieren gerade die von Reittieren begleiteten Götter diese Bewegung: Selene und Helios in der Friesmitte, und noch weiter im Westen Eos.

Gleichzeitig aber sind diese Leitelemente mit statisch-parataktischen Szenen durchmischt: In der Osthälfte wird Phoibes Bewegung nach Westen von Themis abgefangen, die in Richtung Osten kämpft; und schließlich bringen die beiden Zweikampfgruppen mit den Göttern Ouranos and Aither die Bewegung zum Erliegen.

Wiederaufgenommen von Selene wird die Bewegung westwärts dann erneut in der Zweikampfgruppe von Theia angehalten; und nach einem

33 Schrammen (1906) 93-118, bes. 108-118; Radt (1996); Radt (1999) 92-93; Massa Pairault (2007b) 3-4.

34 Auf diese Ausrichtung deutet folgende Inschrift, die wohl die Siege Attalos I. feierte: Fränkel (1890) Nr. 41. - Die drei übereinanderliegenden Fundamente für Denkmäler im WTeil der Oberen Agora dürften damit in Zusammenhang stehen.

35 Vgl. Massa Pairault (2007b) 4.

36 Webb (1998), bes. 244-254; Ridgway (2000) 23-25. 
erneuten Wiederanschieben durch die raumgreifende Gruppe von Helios mit seinem Viergespann endet die westläufige Komposition vollends mit der reitenden Eos: Sie trifft auf den im Worksop-Relief dargestellten Gott, der im Rückenakt dargestellt ist und sich mit seinem Jagdspieß in den stierköpfigen Giganten hineingebohrt hat und der damit die aus der W-Ecke ostwärts drängende Komposition von Rhea auf einem Reitlöwen, einer weiteren Göttin und schließlich einem Giganten mit Doppelhammer und dem Stier-Giganten zum Halten bringt.

Die am S-Fries zu beobachtende Kombination unterschiedlicher Kompositionselemente wirft im Blick aus der Distanz wesentliche Schlaglichter auf die hier gezeigten Götter mit den Reittieren: Rhea, Eos, Helios und Selene. Sie erscheinen deutlich hervorgehoben: Sie sind jeweils verhältnismäßig raumgreifend präsentiert; und dies führt zugleich auch dazu, dass sie nicht in direkter Konfrontation mit einem Gegner erscheinen; jene sind nicht zuletzt durch die Reittiere auf Distanz gehalten. Damit erscheinen die vier Figuren als Ruhepunkte der Komposition.

Die Betonung dieser Götter lenkt die Aufmerksamkeit weg vom Kampfgeschehen und hin auf die Feier göttlicher Erhabenheit. Dies liefert sowohl eine angemessene Orchestrierung für die inhaltliche Ausrichtung der Oberen Agora, die zu Füßen der Altarterrasse liegt, und ebenso aber auch für den Athena-Tempel darüber: Die Götter des Lichts und der Erde - Rhea, Eos, Helios und Selene - verlassen damit aus der Distanzsicht ihren eigentlichen Erzählhorizont, als Teil der Gigantomachie, und treten an, um den Athena-Tempel mit einer Darstellung des Zeitenlaufs zu unterfüttern, wie er sich bisweilen auch in anderen Giebeldekorationen findet, etwa auch dem O-Giebel des Parthenon. ${ }^{37}$ So wäre dann also der Athena-Tempel aus der Sicht von der Oberen Agora in Anspielung auf den berühmten athenischen Tempel gestaltet. ${ }^{38}$

Anders als die direkte Form der Metalepse, wie sie sich an den Risalitinnenseiten des Großen Frieses beobachten lässt, ist die metaleptische Wirkung des Frieses aus der Fernsicht damit nicht mit einer Realitätssteigerung der mythischen Darstellung beschäftigt, sondern zielt vielmehr darauf, den Großen Altar funktionstechnisch in einem zweiten Kontext zu etablieren, und zwar als Basis des Athena-Tempels. Damit handelt es sich

37 Zum O-Giebel des Parthenon s. Berger (1974) 15-16, 19-21; mit einer neuen Rekonstruktion Palagia (1993) 28-30.

38 Umgekehrt erlaubte der Blick hinunter von den Terrassierungen des Abhangs zwischen Altar- und Athena-Terrasse, den N-Fries mit seinen stark parataktisch wirkenden einzelnen Kampfgruppen wahrzunehmen, die auch aus der Distanz die intensive Dynamik des Kampfgeschehens vermittelt haben dürften. - Zur architektonischen Gestaltung des Hangs s. Schrammen (1906) 88-90. 
hier um eine Mischform ontologischer und epistemologischer Metalepse - ontologisch in dem Sinne, dass sie sich klar im Raum manifestiert und nur im Raum funktioniert; und epistemologisch, weil die Darstellungen hier in einen völlig neuen inhaltlichen Bezugsrahmen gestellt werden.

Metaleptische Skalierungen: Der Große Fries aus der Nähe

Die Situation direkter Metalepse an den Risalitinnenwänden und die funktions-oszillierende Form der Metalepse, die sich am S-Fries aus der Fernsicht generiert, bilden die Extreme metaleptischer Ausgestaltung am Großen Fries. Dazwischen lassen sich weitere Formen metaleptischer Diegese verorten, die sich dem Betrachter aus der Nahsicht beim Umschreiten des Baus eröffnen.

Die spezifische Anlage des Altarbaus - die Notwendigkeit für den Besucher, den Bau zunächst zu umschreiten - bedingt, dass die Darstellungen grundsätzlich diegetisch funktionieren: Sie führen den Betrachter durch die Erzählung, während sie ihn zum Altaraufgang begleiten. Die Komposition des Großen Frieses im Raum übernimmt somit die Rolle der Erzählstimme - vergleichbar der Situation auf den Vasen, bei denen der Gefäßkörper zu einer solchen Erzählstimme wird. Zwei Aspekte sind dabei für den Großen Fries aus dem Blickwinkel der Metalepse von besonderem Interesse.

\section{Der Betrachter im Bildraum}

Der Fries ist dank seiner Positionierung für Betrachter vorrangig aus der Untersicht erfahrbar. ${ }^{39}$ Gleichzeitig erscheinen die Figuren durch das extreme Hochrelief und die Abgeschlossenheit des Reliefgrunds den Betrachtern zugewandt: ${ }^{40}$ Die Gestaltung des Reliefs lässt die Figuren optisch in den Raum der Betrachter hineinragen.

Abseits dieser allgemeinen Tendenzen ist jedoch auffällig, dass gerade immer wieder die Leiber der sterbenden bzw. bereits toten Giganten so positioniert sind, dass ihre schmerzverzerrten Gesichter dem Betrachter beim Umschreiten besonders ins Auge fallen. $\mathrm{Zu}$ nennen sind hier etwa am O-Fries die bereits am Boden liegenden Gegner von Apollon und Artemis - ersterer blickt in die Gesichter jener, die von NO heranschreiten;

39 Die Ausnahme bilden die Friesabschnitte, die an den Risalitinnenseiten die Treppe säumen.

40 Kähler (1948) 84-96. 


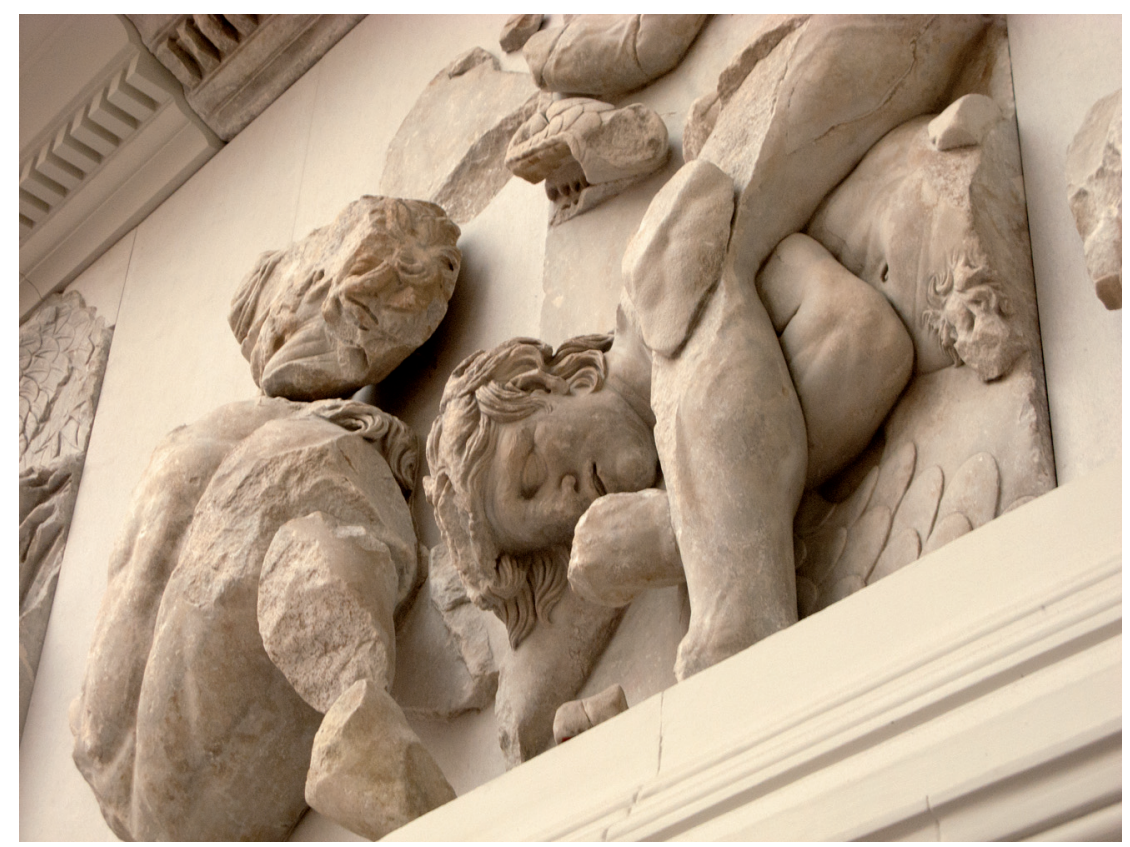

Abb. 4: Der Blick in das Gesicht des Gegners der Phoibe am Südfries. Foto: Autor.

letzterer, der gegen den Molosserhund kämpft, in die aus SW kommenden. Am S-Fries ist der bereits am Boden sich krümmende Gegner der Phoibe zu nennen, der noch versucht, sich eine Lanze aus der Brust zu ziehen - der von der SO-Ecke heranschreitende Betrachter blickt direkt in sein Gesicht (Abb. 4). Am N-Fries schließlich fällt den aus der NO-Ecke kommenden Betrachtern das Gesicht des toten Gegners der Aphrodite ins Auge, aus dessen Brust die Göttin gerade ihren Speer zieht; und dann das Gesicht des Gegners der Moiren, der bereits zu Boden gesunken ist.

Wie bereits in der Darstellung der Gigantomachie aus archaischer Zeit am Siphnier-Fries sind es auch am Großen Fries gerade die Giganten, die in dieser Form dem Betrachter entgegentreten. Und wie in Delphi ist in Pergamon zu beobachten, dass auch die Trabanten der Götter bisweilen den Betrachtern ins Antlitz blicken, so beispielsweise der Hund, der sich am O-Fries zu Füßen von Hekate in ein Schlangenbein verbissen hat, oder jener am S-Fries, der Phoibe und Asteria unterstützt. Diese Form des „Herausschauens“ ist als Akt der Metalepse zu werten, und zwar als eine dezidiert ontologische Metalepse. Dies ist wohlgemerkt keine Form illusionistischer Darstellung, sondern eine Maßnahme, die den Betrachter 
ganz konkret in den Bildraum mit einbezieht und dadurch zur Realitätssteigerung der Darstellung beiträgt. Der Blick in die schmerzverzerrten Züge der Göttergegner und die Aufmerksamkeit von Seiten der Trabanten versetzt die Betrachter erneut in die Rolle der göttlichen Bundesgenossen. Das metaleptische Design fungiert also auch hier als eine Einladung zum Mitkämpfen. Die hier nun eingeforderte Form der Teilnahme aber ist von anderer Qualität als jene, die dem Betrachter auf den Stufen abverlangt wird: Hier ist keine physische Gewalt gefragt, sondern vielmehr eine Art untermalendes Bewundern der Gewalt der Götter, das sich im Leid ihrer Gegner und in der Tatkraft ihrer Trabanten manifestiert.

\section{Der Betrachter im Referenzraum}

Der Große Fries ist im Wesentlichen monoszenisch gestaltet: Die Gigantomachie wird als ein Moment erzählt. Und doch setzen einzelne Aspekte der Komposition andere Akzente und generieren Referenzbeziehungen zwischen einzelnen Teilen des Frieses bzw. zu anderen Monumenten. Dies kann eine Form des vergleichenden Sehens unterstützen, welche durchaus Züge metaleptischen Erzählens trägt. Dieser Prozess lässt sich besonders gut am nördlichen Teil des O-Frieses mit der Darstellung von Zeus und Athena beobachten.

Am O-Fries kämpft Athena zur Rechten gegen den Giganten Alkyoneus, indem sie ihn vom Boden und weg von seiner Mutter Ge zerrt - die einzige Art, ihn zu töten (Abb. 5). Die zur Linken von Athena schwebende Nike zeigt an, dass die Mühen der Göttin sich lohnen werden, denn sie ist dabei, Athena mit einem Kranz als Zeichen des Sieges zu bekrönen. Links von Athena kämpft Zeus gegen drei Giganten: Links von ihm ist ein jugendlicher Gigant zusammengebrochen, dessen Oberschenkel von einem Blitzbündel durchbohrt ist; ein zweiter jugendlicher Gigant ist rechts vor Zeus in die Knie gebrochen und versucht, sich von dem Geschoss zu befreien, das seinen Rücken durchbohrt; ${ }^{41}$ einzig der dritte Gegner, Porphyrion, der bärtige Führer der gesamten Sippe, kämpft weiterhin gegen Zeus, der im Begriff ist, ein weiteres Blitzbündel auf ihn zu schleudern. ${ }^{42}$

41 Simons Deutung ist überzeugend: Dieser Gigant wurde von einem Pfeil des Herakles verwundet (Simon (1975) 18), und nicht von der Aigis des Zeus (so etwa Winnefeld (1910) 53).

42 Zur Zuordnung der Namensinschrift zu dieser Figur s. Kästner (1994) 126-129. 


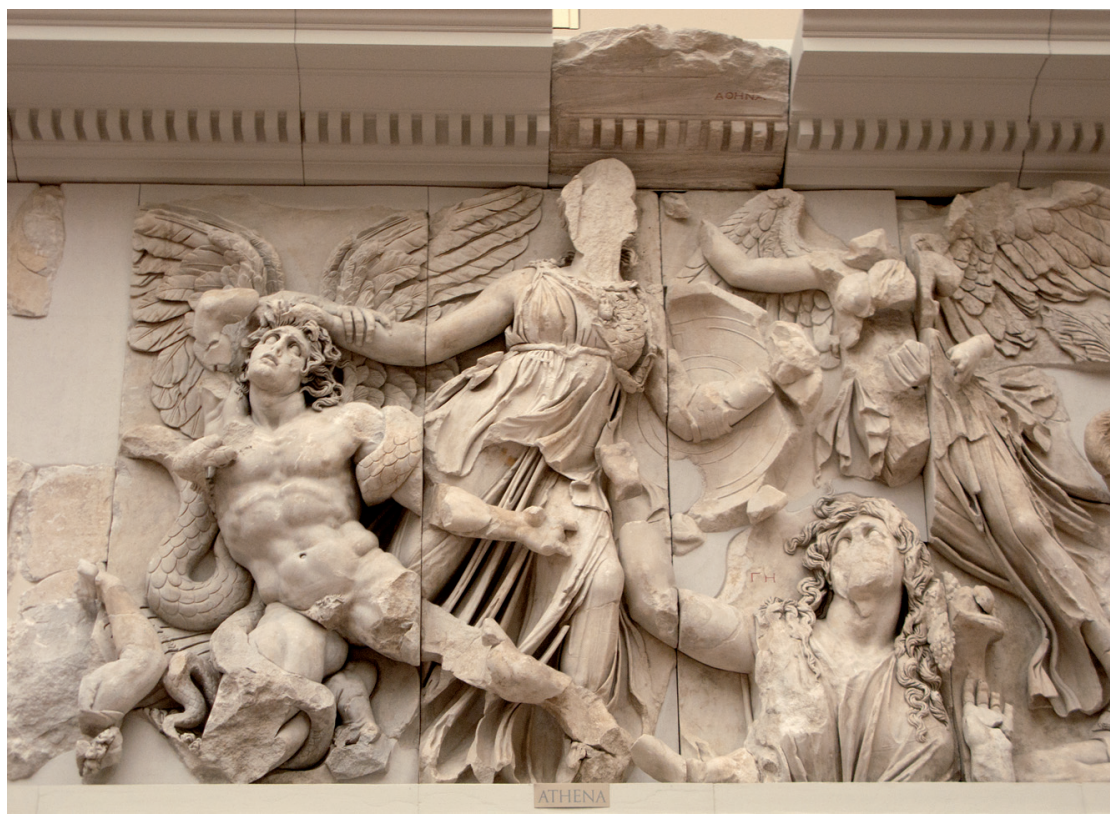

Abb. 5: Athena tötet den Giganten Alkyoneus, indem sie ihn vom Boden und dem Schutz seiner Mutter Ge wegzerrt. Foto: Autor.

Nun findet sich die V-Komposition, in der Athena und Zeus am O-Fries zueinander geordnet sind, erneut an der nördlichen Risalitinnenwand, wo ein weiteres Vater-Tochter-Paar, Okeanos und Doris, im Kampf gegen zwei Giganten zu sehen ist. ${ }^{43}$ Doch gibt es auch Unterschiede in der Charakterisierung der beiden Friesteile: Während Zeus und Athena jeder für sich kämpfen, stehen Okeanos und Doris gemeinsam gegen die Giganten; und während die Olympier gegen insgesamt vier Giganten kämpfen (und ein fünfter zwischen ihnen bereits tot am Boden liegt), begegnen die Meeresgötter gemeinsam mit zwei weiteren Gottheiten nur drei Giganten.

Das Wiederaufgreifen des für Athena und Zeus gewählten Kompositionstypus an späterer Stelle des Frieses stellt eine Verbindung zwischen den beiden Friesteilen her und lädt die Betrachter dazu ein, Vergleiche zwischen den einzelnen Abschnitten anzustellen. In diesem Fall kann so die zuvor betrachtete Leistung der Olympier nochmals als eindrücklicher

43 Zur Wiederholung des Kompositionsschemas s. Kähler (1948) 124-125; vgl. auch Winnefeld (1910) 89; Pfanner (1979) 50-57. - Zuletzt zu diesem Friesabschnitt s. Junker (2003), bes. $435-443$. 
wahrgenommen werden, leisten sie doch ungleich mehr als die Meeresgötter.

$\mathrm{Zu}$ einem metaleptischen Faktor wird das Motiv der V-Komposition aber erst, wenn man es als indexikalischen Verweis auf die athenische Kunst betrachtet. Die Kunst Pergamons ist voll von Rückbezügen auf die klassische Kunst Athens - eine Zeit kultureller Blüte, welcher die Attaliden nacheiferten. ${ }^{44}$ So wurde zunächst argumentiert, dass die Athena am Großen Fries ein direktes Zitat der Darstellung im O-Giebel des Parthenon sei, ${ }^{45}$ eine Annahme, die seit Ernst Bergers neuer Rekonstruktion des Giebels nicht mehr tragfähig ist. ${ }^{46}$ Alternativ wurden die Darstellungen von Zeus und Athena als Bezugnahme auf die Gruppe von Athena und Poseidon im W-Giebel des Parthenon verstanden. ${ }^{47}$ Dort kämpfen die beiden Götter um die kultische Vorherrschaft über Attika, indem sie jeweils ihre Gaben offerieren: Poseidon eine Quelle, Athena den Ölbaum. Die Ähnlichkeit der Komposition macht die Zeus-Athena-Gruppe zum Index des Parthenon-Giebels, und damit ganz allgemein zum Index athenischer Kunst - ein Prozess der ideologischen Positionierung von Seiten der Pergamener. ${ }^{48}$

Relevant für die Schaffung metaleptischen Gehalts sind aber die Unterschiede, die zwischen dem Motiv in Athen und in Pergamon bestehen. Die beiden Götter am Großen Fries kämpfen nicht gegeneinander, sondern gegen einen gemeinsamen Feind: Ganz allgemein ist die Gigantomachie ein Mythos der Restauration, in der gerade das kollektive $\mathrm{Zu}$ sammenwirken der Götter betont wird. ${ }^{49}$ Und weiter: Im Vergleich zum Parthenon-Giebel ist am Großen Fries die Distanz zwischen den beiden

44 So bereits während der ersten Jahre der Ausgrabung attestiert von Wilamowitz-Moellendorff (1881) 159: „Das Hellenische als solches festzuhalten und zur Herrschaft zurückzuführen, das war das Zeichen in dem Pergamon allein siegen konnte und gesiegt hat. Das spezifisch Hellenische ist Athen. Athen war in einer Stunde ähnlicher Gefahr rettend gewesen; an die nationalen Gedanken, welche einst im attischen Reiche, in der attischen Weltsprache, in der attischen Religion sich verkörpert hatten, galt es anzuknüpfen. Dieselbe Göttin, der Pheidias Nike in die Hand gegeben, hält als Nikephoros Wacht auf der pergamenischen Burg. Dieselbe religiöse Geschichtsauffassung, die in dem Aischyleischen Drama und in den Metopen des Parthenon zum Ausdruck kommt, liegt den Kampfgruppen zugrunde, mit denen der Eponymos der Attalis die Südmauer der athenischen Burg ziert, und es ist geschichtlich im höchsten Sinne, daß die Athena der Gigantomachie des großen pergamenischen Altares die Gestalt hat, die ihr ein attischer Künstler in einem attischen Werk gegeben hatte, und daß ihr die Erechtheusschlange den Asterios bezwingen hilft."

45 Dies wurde zuerst bemerkt von Collignon/Pontremoli (1900) 88.

46 Berger (1974) 15-6, 19-21.

47 Winnefeld (1910) 235.

48 Kähler (1948) 118f.

49 Giuliani (2000) 274-277. 
vergrößert, und so wird zwischen ihnen Platz für die toten bzw. sterbenden Gegner geschaffen.

Das Resultat dieser Modifikation ist eine Betonung der durchschlagenden Kraft der Götter, und nicht so sehr ihrer kämpferischen Anstrengung, wie dies am Parthenon der Fall ist. Damit aber wird das motivische Zitat am Großen Fries auch zu einer distinkten Stellungnahme gegenüber dem Vorläufer in Athen: Während dort zwei Götter gegeneinander um die Vorherrschaft kämpfen, sind hier in Pergamon Zeus und Athena gemeinsam mit allen anderen Göttern zusammengekommen, um eine Gefahr von kosmischen Ausmaßen zu besiegen - und diese Kooperation, und auch ihr Erfolg, ist nicht zuletzt auch darin ausgedrückt, dass sich das Motiv der V-Komposition am Altaraufgang nochmals wiederholt.

Der indexikalische Gebrauch des Kompositionsmotivs wird - indem es einen Vergleich mit dem klassischen Athen aufruft, zugleich aber auch dazu genutzt werden kann, sowohl die dargestellte Erzählung als auch Pergamon selbst ideologisch in ganz spezifischer Weise zu positionieren zu einem Werkzeug epistemologischer Metalepse. Die Bildgeschichte wird hier aus ihrer narrativen und räumlichen Verankerung gelöst; und so wird den Betrachtern der Zugriff auf eine weitere, eine abstrakte Erzählebene ermöglicht, auf welcher der Große Altar sowohl als Brückenschlag hin zum Klassischen Athen konzipiert ist ebenso wie als politische Invektive: ${ }^{50}$ ein Siegesmonument, das als Spiegel der militärischen Fähigkeiten der Attaliden verstanden werden kann, modelliert nach Art der großen athenischen mythologischen Schlachtdarstellungen $;{ }^{51}$ und ebenso als selbstbewusste Stellungnahme attalischer Machtansprüche und politischer Strategie, an die das klassische Athen nicht heranreicht.

Der Große Fries und die Metalepse als Kategorie der Bildwissenschaft

Am Großen Fries lassen sich sowohl ontologische als auch epistemologische Formen der Metalepse beobachten: Zum einen greifen die Figuren des Frieses, und vorrangig die Giganten, ganz konkret in die Daseinsebene der Betrachter aus. Dies ist ein Vorgang, der hier gerade durch die architektonische Gestalt des Baus, der den Fries trägt, möglich wird. In diesem Sinn funktioniert der Fries denn auch wie andere rundplastische Werke, und nicht wie die Vasenmalerei.

50 Zu den möglichen Funktionen des Baus s. Webb (1998) 244-250; Kästner (2011).

51 So etwa Kähler (1948), bes. 144-149; Junker (2003). 
Zum anderen sind Formen der Komposition gewählt, welche die Betrachter mit neuen Bezugsrahmen konfrontieren - wie etwa dem Verhältnis von Altar zu Tempel der Athena Polias Nikephoros, oder von Pergamon zu Athen. An diesen Punkten treten ontologische und epistemische Formen der Metalepse vermischt auf - mit einem Resultat, das der Funktionsweise der mehransichtigen Gruppen des 3. Jh.s durchaus vergleichbar ist: Die Bewegung durch den Raum ist sowohl eine Reise durch unterschiedliche Ebenen der Erzählung als auch durch konzeptuelle Bezugsrahmen. Und dies lässt sich dann auch mit der Funktionsweise der spätklassischen Vasen parallelisieren, bei denen der Vasenkörper zum „Erzählkörper“ wird.

So ist denn an diesen Monumenten der Wechsel zwischen unterschiedlichen Diegesen ganz explizit ein Phänomen, das sich im und durch den Raum vollzieht: Die Kontaktzonen zwischen den narrativen Ebenen und zwischen Erzählung und Daseinsebene der Betrachter manifestieren sich im physikalischen Raum und werden so konkret erlebbar. Der einzige Unterschied zwischen den unterschiedlichen Typen von Monumenten - zwischen Flächenkunst und Rundplastik - ist, dass der Raum jeweils unterschiedlich generiert wird.

Die Manifestation der Erzählung im physikalischen Raum garantiert eine gesteigerte Präsenz der Bilddarstellung, gewissermaßen einen Mechanismus zur Steigerung der enargeia der Bilder. ${ }^{52}$ Doch muss dies nicht notwendig bedeuten, dass es auch zu einer Steigerung des Realitätsgehalts der Darstellung kommt. Das Beispiel des Großen Frieses vielmehr zeigt, dass ontologische und epistemologische Formen der Metalepse so miteinander verwoben werden können, dass sie nicht ein realitätsnahes, sondern vielmehr ein durch und durch virtuelles Erlebnis in der Kontaktzone von Monument und Betrachter schaffen. Gerade dieses Charakteristikum hat umfassende Auswirkungen auf die formal-stilistische ebenso wie die bildwissenschaftliche Analyse in der Klassischen Archäologie.

Die Metalepse und die formal-stilistische Bildanalyse

Der Blick auf die metaleptischen Skalierungen erlaubt eine Revision der Entwicklung des Verhältnisses von Bildwerk und Betrachter von der Spätklassik in den Hochhellenismus, etwa auch im Kontrast zu der von Christian Kunze aufgezeigten Entwicklung vom 4. ins 3. Jahrhundert vor Christus. ${ }^{53}$ Nach Kunze sind die Skulpturen der Spätklassik auf eine

52 Zanker (1981); (1987).

53 Kunze (2002) 230f. 
Schaufront hin gestaltet, also klar auf den Betrachter bezogen, machen aber andererseits keine Anstalten, mit dem Betrachter in Kontakt zu treten. ${ }^{54}$ Die Skulpturen des Hochhellenismus hingegen erschließen sich dem Betrachter komplexer: Sie besitzen keine derart direkt zugängliche Schaufront, bieten andererseits aber gerade dadurch dem Betrachter ein intensiveres, dynamischeres Erleben der Darstellung. Zugleich, laut Kunze, erscheinen die Figuren des Hochhellenismus aber vollkommen ungestört in ihren Handlungen, und nicht auf den Betrachter bezogen. Er argumentiert, dass dies den Betrachter in die Rolle eines Zeugen versetzt und damit wiederum der Realitätsanspruch der Darstellung erhöht wird.

Aus dem Blickwinkel der Metalepse, also im Blick auf die Artefaktbzw. Betrachteraktivierung, stellt sich die Situation anders dar. Zwar hat Kunze mit seiner Beobachtung zur Schaufront-Gestaltung der spätklassischen Werke und ihrem Fehlen bei jenen des Hochhellenismus völlig recht, doch zeigt gerade der Fokus auf die metaleptischen Funktionsweisen, dass die Kategorie „Schaufront“ nur ein Aspekt des Bild-Objekt-Verhältnisses ist.

Im Blick auf die Kategorie Metalepse ist stattdessen bereits an den Werken der Spätklassik - und zwar sowohl an der Flächenkunst als auch der Rundplastik - ein deutliches Interesse an der Kommunikation mit dem Betrachter zu beobachten: Hier werden, gerade auch im Spiel mit unterschiedlichen Medien - dem Text und dem Bild - visuelle Ambivalenzen und Betrachtungspfade aufgezeigt, die den Betrachter involvieren sollen. Der tatsächliche qualitative Unterschied zu den Werken des Hochhellenismus liegt damit nicht im generellen Interesse an einer Einbeziehung der Betrachter, sondern vielmehr darin, wie dies umgesetzt ist, denn während bei den spätklassischen Werken ontologische und epistemologische Metalepse nebeneinander zum Einsatz gebracht sind, werden sie in den Werken des Hochhellenismus so ineinander verwoben, dass sie zum gegenseitigen Motor werden und, wie weiter oben beschrieben, nicht die Realität bestätigen, sondern eine virtuelle Erlebniszone schaffen.

\section{Die Metalepse und die bildwissenschaftliche Analyse}

In der klassisch-archäologischen Forschung sind unterschiedliche Formen des Erzählens als charakteristisch für bestimmte künstlerische Epochen angesehen worden, so etwa das zyklische Erzählen für die hellenistische

54 Kunze bezieht sich hier auf die Beobachtungen von Gerhard Rodenwaldt und Adolf Borbein: Rodenwaldt (1944); Borbein (1973). 
Epoche. ${ }^{55}$ Dabei spielte aber keinerlei Rolle, welchen Einfluss die Wahl einer bestimmten Erzählform auf den zu übermittelnden Inhalt hat - die Kategorie der Erzählung wurde als Bildform verstanden, als Maßeinheit zur Bestimmung des Grades, in welchem ein Bild eine naturalistische Wiedergabe einer textlichen Erzählung leistet; sie wurde aber nicht als sinnhaftes Bildmotiv verstanden.

Der Blick auf die Metalepse hilft, hier nachzufassen und vor allem auch Formen der Erzählung gerade mit Blick auf ihre Räumlichkeit zu bedenken; und wie Raum gerade in der Bilderzählung zu einem wesentlichen Faktor werden kann; kurz: wie die Korporalität des Bildes bzw. des Monuments als wesentliche Kategorie für die Analyse von Bilderzählung fruchtbar gemacht werden kann.

Genau hierin liegt meines Erachtens die Stärke einer Sensibilisierung für die Kategorie Metalepse in der klassisch-archäologischen Forschung, und in der Bildwissenschaft ganz allgemein. Dafür ist jedoch entscheidend, so denke ich, die Definition von Metalepse, so wie sie in den Literaturwissenschaften begegnet, zu modifizieren - nämlich dergestalt, dass der Materialträger in die Rolle der Erzählstimme gesetzt wird. Dann - und dies ist aus bildwissenschaftlicher Sicht vielleicht das bemerkenswerteste leistet die aus der Literaturwissenschaft entlehnte Metalepse als analytische Kategorie in der klassisch-archäologischen Forschung eine Fokussierung hin auf die Untersuchung der spezifischen Bildlichkeit der Monumente. Und so wird es eben auch und gerade im Blick auf die hellenistische Flächenkunst möglich, die künstlerische Entwicklung von der Spätklassik bis zum Hochhellenismus neu zu durchdenken.

\section{Bibliographie}

Berger, Ernst: Die Geburt der Athena im Ostgiebel des Parthenon. Basel 1974.

Borbein, Adolf H.: „Die griechische Statue des 4. Jahrhunderts v. Chr. Formanalytische Untersuchungen zur Kunst der Nachklassik“. In: Jahrbuch des Instituts 88 (1973), 43-212.

Brinkmann, Vinzenz: „Die aufgemalten Namensbeischriften an Nord- und Ostfries des Siphnierschatzhauses“. In: Bulletin de Correspondence Hellénique 109 (1985), 771-30.

Burn, Lucilla: The Meidias Painter. Oxford 1987.

Camponetti, Giovanna: „L'hydria londinese di Meidias. Mito e attualità storica ad Atene durante la guerra del Peloponneso". In: Il vasaio e le sue storie. Giornata di

55 S. z.B. Weitzmann (1947); Snodgrass (1981); Giuliani (2003); dazu auch StansburyO’Donnell (1999) 1-17. - Für den Großen Fries s. z.B. v. Salis (1912), bes. 150-153. 
studi sulla ceramica attica in onore di Mario Torelli per i suoi settanta anni. $\mathrm{Hg}$. v. Simonetta Angiolillo u. Marco Guiman. Cagliari 2007, 17-45.

Collignon, Maxime u. Emmanuel Pontremoli: Pergame. Restauration et description des monuments de l'Acropole. Paris 1900.

Dreyfus, Renée u. Ellen Schraudolph: Pergamon: The Telephos Frieze from the Great Altar 1-2. San Francisco 1996-97.

Fludernik, Monika: „Scene Shift, Metalepsis, and the Metaleptic Mode“. In: Style 37 (2003), 382-400 (= „Changement de scène et mode métaleptique“. In: Métalepses. Entorses au pacte de la représentation. Hg. v. John Pier u. Jean-Marie Schaeffer. Paris 2005, 73-94).

Fränkel, Max: Die Inschriften von Pergamon 1: Bis zum Ende der Königszeit. Altertümer von Pergamon VIII.1. Berlin 1890.

Genette, Gerard: Narrative Discourse: An Essay in Method. Ithaca 1983.

Genette, Gerard: Métalepse: de la figure à la fiction. Paris 2004.

Giuliani, Luca: „Die Giganten als Gegenbilder der attischen Bürger im 6. und 5. Jahrhundert v. Chr.“. In: Gegenwelten zu den Kulturen Griechenlands und Roms in der Antike. Hg. v. Tonio Hölscher. München, Leipzig 2000, 263-286.

Giuliani, Luca: Bild und Mythos. München 2003.

Hoepfner, Wolfram: „Zu den großen Altären von Magnesia und Pergamon“. In: $A r$ chäologischer Anzeiger (1989), 601-634.

Hoepfner, Wolfram: „Siegestempel und Siegesaltäre. Der Pergamonaltar als Siegesmonument". In: Die griechische Polis. Architektur und Politik. Hg. v. Wolfram Hoepfner u. Gerhard Zimmer. Tübingen 1993, 111-125.

Hölscher, Tonio: „Die Geschlagenen und Ausgelieferten in der Kunst des Hellenismus“. In: Antike Kunst 28 (1985), 120-136.

Jong, Irene de: „Metalepsis in ancient Greek literature“. In: Narratology and Interpretation: The Content of Narrative Form in Ancient Literature. Hg. v. Jonas Grethlein u. Antonios Rengakos. Berlin, New York 2009, 87-116.

Junker, Klaus: „Meerwesen in Pergamon. Zur Deutung des Großen Frieses“. In: Mitteilungen des Deutschen Archäologischen Instituts, Abteilung Istanbul 53 (2003), 425-443.

Kähler, Heinz: Der große Fries von Pergamon. Berlin 1948.

Kästner, Volker: „Gigantennamen“. In: Mitteilungen des Deutschen Archäologischen Instituts, Abteilung Istanbul 44 (1994), 125-134.

Kästner, Volker: „Die Altarterrasse“. In: Pergamon. Panorama der antiken Metropole. Begleitbuch zur Ausstellung. Hg. v. Ralf Grüssinger, Volker Kästner u. Andreas Scholl. Petersberg 2011, 198-211.

Kemp, Wolfgang: „Ellipsen, Analepsen, Gleichzeitigkeiten. Schwierige Aufgaben für die Bilderzählung". In: Der Text des Bildes. Möglichkeiten und Mittel eigenständiger Bilderzählung. Hg. v. Wolfgang Kemp. München 1989, 62-88.

Krahmer, Gerhard: „Stilphasen der hellenistischen Plastik“. In: Mitteilungen des Deutschen Archäologischen Instituts, Abteilung Rom 38/39 (1923/24), 138-184.

Krahmer, Gerhard: „Nachahmungen des 5. Jahrhunderts in Pergamenischen Statuen“. In: Mitteilungen des Deutschen Archäologischen Instituts, Abteilung Rom 40 (1925), 67-106.

Künzl, Ernst: Frühhellenistische Gruppen. Köln 1968.

Luca, Goia de u. Wolfgang Radt: Sondagen im Fundament des Großen Altars. Pergamenische Forschungen 12. Berlin 1999. 
Kunze, Christian: Der Farnesische Stier und die Dirkegruppe des Apollonios und Tauriskos. Jahrbuch des Deutschen Archäologischen Instituts. Ergänzungsheft 30. Berlin, New York 1998.

Kunze, Christian: Zum Greifen Nah. Stilphänomene in der hellenistischen Skulptur und ihre inhaltliche Interpretation. München 2002.

La Rocca, Eugenio: „Die Zwölf Götter, Hera und die Verherrlichung der Attaliden am großen Altar von Pergamon“. In: Jahrbuch der Berliner Museen 40 (1998), 7-30.

Linfert, Andreas: „Prunkaltäre“. In: Stadtbild und Bürgerbild im Hellenismus. Hg. v. Michael Wörrle u. Paul Zanker. München 1995, 131-146.

Lorenz, Katharina: „The anatomy of metalepsis: visuality turns around on late fifthcentury pots". In: Debating the Athenian Cultural Revolution. Art, literature, philosophy and politics 430-380 B.C. Hg. v. Robin Osborne. Cambridge 2007, 116-143.

Lorenz, Katharina: Mythological Images and their Interpretation. Iconology, Semiotics and Image Studies in Ancient Art History. Cambridge [in Vorbereitung].

Mahne, Nicole: Transmediale Erzähltheorie. Eine Einführung. Göttingen 2007.

Manakidou, Flora: Beschreibungen von Kunstwerken in der hellenistischen Dichtung. München 1993.

Marvin, Miranda: „The Ludovisi barbarians: The grand manner“. In: The Ancient Art of Emulation: Studies in Artistic Originality and Tradition from the Present to Classical Antiquity. Hg. v. Elaine K. Gazda. Ann Arbor 2002, 205-223.

Massa Pairault, Françoise-Hélène: „L'interprétation des frieses du Grand Autel de Pergame et des stylopinakia de Cyzique. Quelques problèmes“. In: Images et modernité hellénistiques. Appropriation et représentation du monde d'Alexandre à César. Hg. v. Françoise-Hélène Massa Pairault u. Gilles Sauron. Rom 2007, 205-221 [= Massa Pairault (2007a)].

Massa Pairault, Françoise-Hélène: La Gigantomachie de Pergame ou l'image du monde. Athen 2007 [= Massa Pairault (2007b)].

Muth, Susanne: Gewalt im Bild. Berlin 2008.

Neer, Richard: „Framing the gift. The politics of the Siphnian treasury at Delphi“. In: Classical Antiquity 20 (2001), 273-336.

Neer, Richard: The Emergence of the Classical Style. Chicago 2011.

Palagia, Olga: The Pediments of the Parthenon. Leiden 1993.

Pfanner, Michael: „Bemerkungen zur Komposition und Interpretation des großen Frieses von Pergamon“. In: Archäologischer Anzeiger (1979), 46-57.

Queyrel, François: L'Autel de Pergame. Images et pouvoir en Grèce d'Asie. Paris 2005.

Radt, Wolfgang: „Pergamon. Vorbericht über die Kampagne 1995“. In: Archäologischer Anzeiger (1996), 443-454.

Radt, Wolfgang: Pergamon. Geschichte und Bauten einer antiken Metropole. Darmstadt 1999.

Rheidt, Klaus: „Die Obere Agora. Zur Entwicklung des hellenistischen Stadtzentrums von Pergamon". In: Mitteilungen des Deutschen Archäologischen Instituts, Abteilung Istanbul 42 (1992), 235-285.

Ridgway, Brunilde S.: Hellenistic Sculpture II: The Styles of c. 200-100 B.C. Madison 2000.

Rodenwaldt, Gerhardt: „Theoi Rheia Zoontes“. In: Abhandlungen der Berliner Akademie der Wissenschaften 13 (1943).

Ryan, Marie-Laure: „Logique culturelle de la métalepse, ou la métalepse dans tous ses états“. In: Métalepses. Entorses au pacte de la représentation. Hg. v. John Pier u. Jean-Marie Schaeffer. Paris 2005, 201-223. 
Ryan, Marie-Laure: Avatars of Story. Minneapolis 2006.

Scholl, Andreas: „Olympiou endothen aule - Zur Deutung des Pergamonaltars als Palast des Zeus". In: Jahrbuch des Deutschen Archäologischen Instituts 124 (2009), 251-278.

Scholl, Andreas: „Der Pergamonaltar - Ein Zeuspalast mit homerischen Zügen?“. In: Pergamon. Panorama der antiken Metropole. Begleitbuch zur Ausstellung. Hg. v. Ralf Grüssinger, Volker Kästner u. Andreas Scholl. Petersberg 2011, 212-218.

Schrammen, Jakob: Der große Altar, der obere Markt. Altertümer von Pergamon III.1. Berlin 1906.

Schraudolph, Ellen: „Beispiele hellenistischer Plastik der Zeit zwischen 190 und 160 v. Chr." In: Die Geschichte der antiken Bildhauerkunst III: Hellenistische Plastik. Hg. v. Peter Cornelis Bol. Mainz 2007, 189-239.

Simon, Erika: Pergamon und Hesiod. Mainz 1975.

Simon, Erika: „Ikonographie und Epigraphik. Zum Bauschmuck des Siphnierschatzhauses in Delphi“. In: Zeitschrift für Papyrologie und Epigraphik 57 (1984), 1-22.

Snodgrass, Anthony: Narration and Allusion in Archaic Greek Art. Cambridge 1981.

Stähler, Klaus: „Überlegungen zur architektonischen Gestalt des Pergamonaltares“. In: Studien zur Religion und Kultur Kleinasiens. Festschrift Friedrich Karl Dörner I. Hg. v. Sençer Sahin, Elmar Schwertheim u. Jörg Wagner. Leiden 1978, 838-867.

Stansbury-O'Donnell, Mark: Pictorial Narrative in Ancient Greek Art. Cambridge 1999.

Vian, Francis: Répertoire des Gigantomachies figurées dans l'art grec et romain. Paris 1951.

Webb, Pamela A.: „The functions of the sanctuary of Athena and the Pergamon altar

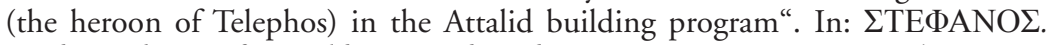
Studies in honor of Brunilde Sismondo Ridgway. Hg. v. Kim J. Hartswick u. Mary C. Sturgeon. Philadelphia 1998, 241-254.

Weitzmann, Kurt: Illustrations in Roll and Codex. A Study of the Origin and Method of Text Illustration. Princeton 1947.

Wilamowitz-Moellendorff, Ulrich von: Antigonos von Karystos. Berlin 1881.

Winnefeld, Herrmann: Die Friese des großen Altars. Altertümer von Pergamon III.2. Berlin 1910.

Zanker, Grahame: Realism in Alexandrian Poetry: A Literature and its Audience. London 1987.

Zanker, Grahame: Modes of Viewing in Hellenistic Poetry and Art. Madison 2004.

Zanker, Grahame: Herodas, Mimiambs. Oxford 2009. 
